\title{
Effects of thermal treatments on protein adsorption of Co-Cr-Mo ASTM-F75 alloys
}

${ }^{1}$ Duncan, L A; ${ }^{1}$ Labeed, F H; ${ }^{1}$ Abel, M-L; ${ }^{2}$ Kamali, A; ${ }^{1}$ Watts, J F

${ }^{1}$ Faculty of Engineering and Physical Sciences, University of Surrey, Guildford, Surrey, U.K., GU2 7XH

${ }^{2}$ Implant Development Centre, Smith \& Nephew Orthopaedics Ltd, Leamington Spa, Warwick, U.K., CV31 3HL

Email: J.Watts@surrey.ac.uk 


\section{$\underline{\text { Abstract }}$}

High mechanical demands are placed upon materials used in hip arthroplasty. Postmanufacturing thermal treatments are commonly employed to reduce shrinkage voids which can occur in as cast alloys. Several studies have investigated the consequences of these treatments upon the alloy microstructure and tribological properties but none to determine if there are any biological ramifications.

In this study the adsorption of proteins from foetal bovine serum (FBS), on three CoCr-Mo ASTM-F75 alloy samples with different metallurgical histories, has been studied as a function of protein concentration. The samples used were in the as cast, solution annealed, and hot isostatically pressed and solution annealed metallurgical condition. Adsorption isotherms have been plotted using the surface concentration of nitrogen as a diagnostic of protein uptake as measured by $\mathrm{x}$-ray photoelectron spectroscopy. The level of protein coverage seen in the three samples is dependent upon the metallurgical history of the sample. The data is shown to be a good fit to the Langmuir adsorption isotherm. Differences in the way protein adsorption occurs on very similar alloys has been observed with the as cast alloy showing slightly different characteristics to the alloys which received subsequent heat treatments. This suggests that development of the tissue/implant interface, although similar, may differ between AC and heat treated samples.

Keywords - Protein adsorption, Adsorption, XPS (x-ray photoelectron spectroscopy), Interface, Cobalt alloy, Plasma proteins. 


\section{Introduction}

High demands are placed on materials used in resurfacing hip arthroplasty. The materials used must possess favourable mechanical properties, high corrosion resistance, wear resistance and be non-toxic. Co-Cr-Mo alloys are one of the most commonly used materials for hip arthroplasty. This alloy is used in a variety of conditions which are dependent upon the composition (e.g. high and low carbon), manufacturing methods (casting, hot forging (wrought) or powder metallurgy), and lastly post-manufacturing thermal treatments, which are time and temperature dependent [1]. High carbon AC Co-Cr-Mo alloys are biphasic containing a matrix rich in cobalt, chromium, and molybdenum and a carbide phase rich in chromium, molybdenum and carbon. The as cast (AC) alloy microstructure contains large blocky carbides and has an overall carbide volume fraction of $~ 5 \%$ [1]. AC Co-Cr-Mo alloys may contain defects known as shrinkage voids which can reduce the overall mechanical properties of the material under certain loading conditions. Thermal treatments such as hot isostatic pressing (HIP) and solution annealing (SA) have been employed to fully densify and therefore improve the mechanical properties of the alloy. In addition the post manufacturing thermal treatments also render the alloy easier to machine [2].

The HIPing parameters of AC Co-Cr-Mo alloys subject the component to a temperature of $1200^{\circ} \mathrm{C}$ for four hours in an inert atmosphere. The components are then quenched in argon at a rate of approximately $8-10^{\circ} \mathrm{C}$ per minute. This process is carried out at an isostatic pressure of approximately $103 \mathrm{MPa}$. The temperature used is close enough to the solidus temperature $\left(\sim 1230^{\circ} \mathrm{C}\right)$ of the alloy to allow the dissolution of chromium, molybdenum and carbon from the carbide phase into the surrounding matrix. As the component is allowed to cool reprecipitation of the carbide phase occurs predominantly at the grain boundaries. This results in a reduction of the overall volume fraction of the carbide phase (approximately 2.3\%), and the formation of finer agglomerated and lamellar carbides. 
The carbide phase is less mechanically stable than in the AC microstructure [2]. Post HIPing the mechanical properties of the alloy can be below those required. SA provides a means of restoring the mechanical properties of the alloy due to the faster cooling of the alloy which provides a generally finer microstructure. SA is also employed to dissolve secondary phases to produce maximum homogeneity.

The procedure for SA of Co-Cr-Mo alloys is similar to that of HIPing however after heating for 4 hours at $1200^{\circ} \mathrm{C}$ there is a rapid quenching of the component to $800^{\circ} \mathrm{C}$ in less than 8 minutes $\left(50^{\circ} \mathrm{C}\right.$ per minute). The process is carried out in an inert atmosphere to prevent oxidation but unlike HIPing this is not carried out under high pressure. As with HIPing the temperatures used cause the diffusion of chromium, molybdenum and carbon from the carbide phase into the matrix. The consequence of the rapid quenching restricts the reprecipitation of the carbide phase. Once a Co-Cr-Mo alloy has been heat treated any subsequent thermal treatments dramatically reduces the overall volume fraction of the carbide phase [2].

As a result of such variations in thermal processing Co-Cr-Mo alloys are able to have a variety of microstructures with the same composition. Tribological studies have shown that lower levels of wear exhibited by AC Co-Cr-Mo alloys has been attributed to their higher overall volume fraction of carbides $[1,3]$. Much work has been carried out to investigate the consequences of thermal treatments upon the mechanical/tribological characteristics of an alloy, but little has been done to study what, if any, biological ramifications exist as a result of metallurgical conditions.

Materials interact with their environment through their interfaces. The development of the tissue/implant interface is crucial in determining the ultimate biological response to the implant [4]. The development of the tissue/implant interface is affected by numerous factors including material, shape, topography and surface chemistry, not to mention patient 
variability [5]. It is widely acknowledged that the initial event that occurs when an implant is introduced into a patient is the adsorption of proteins from the surrounding blood and tissue fluids. The process of protein adsorption is a dynamic one whereby proteins go through conformational changes, denature, or can be replaced by larger proteins; the phenomenon is known as the "Vroman Effect". As the tissue/implant interface develops, cells eventually reach the surface; at this time they are presented not with the surface of the implant but with a proteinaceous layer to which they bind via membrane receptors [6]. The type and conformation of the proteins will determine which cells bind to the site. This will then determine the ultimate biological response to the implant. The protein conditioning layer acts as a precursor to cellular adhesion and establishes the connection between the way in which proteins respond to the surface of the implant and the ultimate tissue response.

This study investigates the adsorption of proteins from foetal bovine serum (FBS) on a Co-Cr-Mo alloy manufactured to the following specifications stipulated in ASTM-75. Three samples, each with a different post-manufacturing thermal history, have been used: AC, SA, and HIPSA.

In the present study, the aim is to investigate the adsorption of proteins from FBS on Co-Cr-Mo ASTM-F75 alloy specimens to determine if post-manufacturing thermal treatments of an implanted material have any influence on the way protein adsorption occurs. Differences in the way in which proteins adsorb onto the surface, if they exist, would suggest that the development of the tissue/implant interface is dependent upon the metallurgical history of the alloy. Furthermore, as the tissue/implant interface develops, the way in which the alloy integrates with tissues is influenced by post-manufacturing thermal treatments.

\subsection{Adsorption and fractional coverage}


In surface science fractional coverage, $\sigma$, is used to express the quantity of material adsorbed onto a surface as a function of complete monolayer coverage. This is the ratio between the number of occupied adsorption sites $(N)$ to the total number of sites available for adsorption $\left(N_{s}\right)$. In terms of classical surface adsorption theory it is usual to consider gas phase adsorption (at constant pressure); fractional coverage can then be described by the ratio between the volume of gas adsorbed $(V)$ relative to the volume of gas adsorbed at monolayer coverage $\left(V_{m}\right)[7]$.

$$
\sigma=\frac{N}{N_{s}}=\frac{V}{V_{m}}
$$

More recently adsorption from the liquid phase has been studied by constructing adsorption isotherms by measuring the amount of material retained on a surface once removed from the liquid solution. The use of XPS in this way was pioneered by Castle \& Bailey [8] and was subsequently extended to ToF-SIMS by Abel et al. [9]. The experimental requirements of this approach have been reviewed by Watts \& Castle [10].

In this work XPS has been used to calculate the fractional coverage based on the surface concentration (atomic \%) of an element diagnostic of the adsorbate. To measure protein adsorption it was assumed that the presence of nitrogen was indicative of protein uptake [11]. Chromium was used as the diagnostic substrate marker to establish the XPS signal attenuation as the adsorbed protein overlayer thickness increased. Fractional coverage was determined by quantifying the XPS spectrum and plotting the surface concentration of the adsorbate (relative to monolayer coverage) as a function of FBS concentration; this is the liquid phase/XPS version of the well known gas phase adsorption isotherm. 


\subsection{Adsorption isotherms}

Adsorption is commonly described using isotherms which indicate the amount of adsorbate retained on the substrate surface as a function of solution concentration, at a constant temperature. The kinetics of adsorption can also be studied, and consideration of both provides an indication of the kinetics and thermodynamics of the adsorption process. In practise it is usual to establish the position of kinetic equilibrium and then conduct the thermodynamic investigations.

Adsorption isotherms give useful information regarding coverage as well as information on the mechanism of adsorption [11]. Adsorption can be achieved through either chemisorption or physisorption, which depends upon the type of bond formed between the adsorbate and the adsorbent (substrate).

Physisorption is due to van der Waals forces which are generally long range but weak. The enthalpy of adsorption is considered to be less exothermic than $-25 \mathrm{kJmol}^{-1}$. Chemisorption is characterised by a stronger interaction between the adsorbate and adsorbent. Stronger secondary and even primary bonds (ionic, metallic, or covalent) are formed, generally with an enthalpy of adsorption more negative than $-40 \mathrm{kJmol}^{-1}$ [10]. An important difference between the two modes of adsorption is their response to heat. Increasing the temperature will lead to a decrease in the amount of material physisorbed, whereas in the case of chemisorption an increase in adsorption will be observed as it is an activated process. Another significant difference between physical and chemical adsorption is related to the saturation level of the adsorbed species. Chemisorption can only ever achieve monolayer formation as adsorption ceases when the adsorbate can no longer make direct contact with the adsorbent. However, physisorption has no such restriction and so is able to form multilayers of many molecules thick. It is possible for an initial chemisorbed layer to act as a substrate for further material to be physisorbed as described by the well-known BET isotherms. 
There are many models describing chemisorption, each with different assumptions used to derive the expressions for surface coverage. Commonly used isotherms include the Langmuir, Temkin and Freundlich isotherms.

The Langmuir isotherm is the simplest isotherm and is based on the following assumptions:

- Multilayer coverage is not possible.

- All adsorption sites are equivalent.

- The probability of the adsorption of a molecule is independent of neighbouring sites being occupied or otherwise.

- The enthalpy of adsorption remains constant.

Using these assumptions the fractional monolayer coverage, $(\sigma)$, in gas phase adsorption studies at pressure, $(\mathrm{P})$, can be expressed as follows:

$\sigma=\frac{b P}{1+b P}$

where $\mathrm{b}$ is the ratio of the rate constants for adsorption and desorption and is related to both the enthalpy of adsorption and temperature. The constant $b$ is usually expressed as:

$b=b_{0} \exp (Q / R T)$

where $b_{0}$ is a frequency factor, $\mathrm{Q}$ is the interaction energy, $\mathrm{R}$ is the gas constant and $\mathrm{T}$ is the temperature. Replacing $\sigma$ with $V / V_{m}$ (equation 1), equation 2 can be rearranged to give: 
$\frac{P}{V}=\frac{1}{b V_{m}}+\frac{P}{V_{m}}$

The Temkin and Freundlich isotherms are based on the theory that the more energetically favourable sites (those with a more negative enthalpy of adsorption) are occupied first by the adsorbate. The Temkin isotherm assumes that adsorption enthalpy changes linearly with coverage. The Temkin isotherm is represented as:

$\sigma=c_{1} \ln \left(c_{2} P\right)$

where $c_{1}$ and $c_{2}$ are constants. The Freundlich isotherm assumes that the adsorption enthalpy varies logarithmically with gas pressure and is given by:

$\sigma=c_{1} P^{1 / c_{2}}$

Adsorption isotherms are expressed in the present study as the amount of adsorbate on the adsorbent as a function of solution concentration. For liquid phase adsorption measured using XPS the Langmuir adsorption equation can be expressed as:

$\frac{c}{x}=\frac{1}{b \Gamma_{m}}+\frac{c}{\Gamma_{m}}$

where $\mathrm{c}$ is the solution concentration, $\mathrm{x}$ is the uptake as measured by XPS (atomic \%) and $\Gamma_{m}$ is monolayer coverage i.e. the maximum uptake as measured by XPS (atomic \%). The test 
of conformity of experimental data to the Langmuir isotherm is achieved by plotting $c / x$ against $x$. Compliance to the isotherm will result in a straight line with a gradient of $1 / \Gamma_{m}$ and an intercept of $1 / \mathrm{b} \Gamma_{m}$. For liquid phase adsorption the Temkin equation 5 becomes:

$\sigma=b \ln c$

and a plot of $\mathrm{x}$ against lnc will exhibit a straight line to show its conformity. Finally, the Freundlich isotherm is expressed as:

$\sigma=b c^{1 / c_{2}}$

Testing the conformity of experimental data to the Freundlich is achieved via a plot of $\log x$ against $\log$. If the data obeys the Freundlich isotherm, a straight line will result.

Although there are many isotherms, the Langmuir and Temkin have been shown to be the most widely applicable in determination of adsorption isotherms by means of surface analysis [10].

\subsection{Materials and methods}

\subsection{Specimen preparation}

As previously stated the three Co-Cr-Mo ASTM-F75 alloy samples included an AC sample, the second sample had undergone SA post-manufacture; the third had been treated by HIPing followed by SA; HIPSA. Coupons were cut from the samples with a $10 \mathrm{~mm}$ diameter and $2 \mathrm{~mm}$ thickness. Samples were polished to provide an unblemished mirror finish using a water based colloidal silica suspension with an average particle size of $0.04 \mu \mathrm{m}$. The polished 
coupons were then cleaned in an ultrasonic bath with deionized water, methanol and, finally acetone for 5 minutes each.

\subsection{Foetal bovine serum}

Protein solutions were prepared using foetal bovine serum (FBS) (Sigma-Aldrich, Poole, UK) with a total protein content of between 4.0-4.3 $\mathrm{gdL}^{-1}$. Solutions were made up by diluting the FBS with deionized water. A series of eight FBS concentrations varying from 100 to $10^{-5} \% \mathrm{v} / \mathrm{v}$ were used. The coupons (once clean) were then placed in $5 \mathrm{ml}$ of the protein solution for 30 minutes to allow sufficient time for adsorption to occur. Subsequently the coupons were removed from the FBS solution and were gently rinsed using Milli-Q water to ensure that any unbound proteins were removed. The same batch of FBS was used to ensure the quantity of proteins in solution was kept constant.

\subsection{X-ray photoelectron spectroscopy}

XPS analysis was conducted using a modified VG Scientific ESCALAB MkII electron spectrometer equipped with a Thermo Alpha 110 electron energy analyser and a Thermo XR3 digital twin anode X-ray source. The twin anode was operated using Al Ka Xray radiation at $300 \mathrm{~W}$. Survey spectra over $0-1350 \mathrm{eV}$ were obtained using a pass energy of $50 \mathrm{eV}$ while high resolution spectra were obtained using a pass energy of $20 \mathrm{eV}$. The control of the spectrometer and subsequent data processing was carried out using the manufacturers software, Avantage (Ver. 4.37). For quantification, a Shirley background was subtracted from the spectra. To reduce damage due to the radiant heat from the twin anode to the delicate adsorbed proteinaceous layer the x-ray source was operated $30 \mathrm{~mm}$ from the sample surface. For a given FBS concentration three identical samples were prepared and analysed in order to 
ensure repeatability. The average value obtained from three samples was used to plot the adsorption isotherms.

\subsection{Results and discussion}

Figure 1 shows the XPS survey spectrum from a polished AC Co-Cr-Mo ASTM-F75 sample. As can be seen the spectrum is dominated by strong signals originating from $\mathrm{C}, \mathrm{O}$, Cr, Co, and Mo.

Quantitative analysis has been carried out to determine the surface composition of the elements present in all three samples' oxide layers, the results of which are presented in Table 2.

The surface composition of all three samples have been found to be similar, with the most abundant species observed on the sample surfaces being carbon and oxygen. The significant surface concentration of carbon is adventitious contamination which may have come from the cleaning process or adsorbed from the ambient. The shape of the high resolution spectra of the Cr2p3/2 (shown in Figure 2) is composed of two components; the first at a binding energy of approximately $574 \mathrm{eV}$ originates from the metallic chromium, the second at a binding energy of $577 \mathrm{eV}$ originates from chromium oxide [12]. The alloys' passive chromium oxide surface accounts for the high levels of oxygen detected.

As previously mentioned, the presence of nitrogen was assumed to be indicative of the presence of adsorbed proteins. Nitrogen is absent in the spectra taken from all three polished and clean samples. However, as shown in Figure 3, it is present in the XPS survey spectrum taken from an AC sample immersed in 100\% v/v FBS for 30 minutes.

Figure 4 shows the high resolution spectra of the N1s peaks after immersion in various FBS concentrations on an arbitrary intensity scale. This shows how the N1s peak increases in intensity with increasing FBS concentrations. 
The concentrations of both nitrogen and chromium on the three Co-Cr-Mo ASTMF75 sample surfaces immersed in increasing FBS strengths are shown in Figures 5, 6, and 7 respectively.

The data shows that a plateau is reached for all three samples (for both nitrogen and chromium) and protein uptake no longer increases as a function of FBS concentration. At this point, monolayer coverage has been reached, indicating that chemisorption has occurred. The point at which a monolayer develops is known as the critical saturation of protein, and is approximately $10 \%$ v/v FBS for all three samples. The nitrogen and chromium isotherm pairs for each of the three samples are in good agreement with one another - this is verified by the fact that all three nitrogen and chromium isotherm pairs indicate monolayer coverage at the same concentration.

Fractional coverage based on the surface concentration of nitrogen is shown for all three samples in Figure 8. The use of a logarithmic scale enables the data points at the low concentrations of the isotherm to be examined and compared. All three samples begin at a similar level of coverage and as the FBS concentration is increased develop higher levels of protein coverage. Pre monolayer coverage, the AC sample shows higher levels of protein uptake than either of the heat treated samples. The two heat treated samples both show remarkably similar levels of coverage over the concentration range used. As can be seen the AC sample reaches near monolayer coverage at much lower FBS concentrations (approx. $0.1 \% \mathrm{v} / \mathrm{v})$.

The estimation of coverage based on the surface concentration of chromium is shown for all three samples in Figure 9. The AC sample shows a higher level of coverage than both the SA and HIPSA samples. As before, the SA and HIPSA samples are showing similar levels of coverage over the concentration range used. 
From the lowest concentration used up to $10 \%$ v/v FBS (the concentration at which critical protein saturation occurs) the experimental data for all three samples has been tested for its conformity to the Langmuir, Temkin, and Freundlich isotherms. The isotherm plots were fitted with a linear line and the least square regression values $\left(\mathrm{R}^{2}\right)$ was obtained. The least square values for the isotherms are compared in Table 3.

As indicated by the $\mathrm{R}^{2}$ values, the experimental data gives a better fit to the Langmuir model than the Temkin or the Freundlich models. As previously stated, the assumptions of the Langmuir model state that the proteins are adsorbed on the Co-Cr-Mo ASTM-F75 alloy as a monolayer, all adsorption sites are equal, and the enthalpy of adsorption is constant.

The Langmuir isotherm constants have been determined and are given in Table 4. The $b$ (Eq. 4) constant is a dimensionless quantity which is indicative of the heat of adsorption and this can be taken as an indication of the strength of interaction between the FBS proteins and the Co-Cr-Mo ASTM-F75 substrates [9], [13]. The b value for the AC sample was found to be approximately 33 times higher than that for the SA sample and approximately 20 times higher than for the HIPSA sample. This suggests that the bonds formed by the proteins present in FBS are stronger on an AC substrate rather than on a heat treated one.

The knee of the isotherms, shown in Figure 10, also gives a qualitative indication of the interaction energy Q between the protein and the substrate [14], related to the Langmuir constant $b$. The knee is much sharper in the isotherm, representing protein uptake on the AC sample. This suggests that the interaction between the proteins and adsorption sites on the AC sample are stronger than those present between the proteins and adsorption sites on heat treated samples. The knees on the SA and HIPSA are both very similar. It is interesting to note that the effect of HIP does not seem to significantly alter the level of coverage seen, or the interaction energy between the proteins and the heat treated substrates. 
The experimentally derived values of maximum coverage $(\Gamma \mathrm{m})$, Table 4 , are in good agreement to those determined using the constants derived from the plots showing Langmuir conformity. This shows the applicability and accuracy of the Langmuir model to the experimental data.

The experimental data suggests that the application of post-manufacturing thermal treatments has an influence upon the adsorption of proteins on Co-Cr-Mo ASTM-F75 alloys. It is therefore possible that the way in which the three samples integrate with tissue are different. It also suggests that a different tissue/implant interface can be achieved on samples which have an identical chemistry and surface finish. The question of which of these alloys would achieve a superior tissue/implant interface will require further investigation.

Some studies [15-17] suggest that adsorbed proteins on the articulating surfaces of hip replacements act as a solid lubricant which is beneficial to the wear of the components. Higher levels of coverage and stronger protein/substrate interactions as seen on the AC sample would suggest that the adsorbed proteinaceous layer could be a contributing factor in the lower levels of wear seen in AC Co-Cr-Mo ASTM-F75 alloys.

\subsection{Conclusions}

Adsorption of proteins from FBS onto Co-Cr-Mo ASTM-F75 has been studied using XPS. Adsorption occurs by way of chemisorption and has been shown to obey the Langmuir model. This indicates the adsorption of a protein monolayer with a constant heat of adsorption. The bonds formed by proteins on an AC Co-Cr-Mo ASTM-F75 substrate are significantly stronger than those formed by proteins onto heat treated Co-Cr-Mo ASTM-F75 alloys.

Development of the protein conditioning layer has been shown to vary on three identical Co-Cr-Mo ASTM-F75 alloys with different metallurgical histories. This suggests 
that the development of the implant/tissue interface may potentially be different for all three samples; the way in which the tissue integrates with the three alloys may therefore be different. This work warrants further investigation with other tissue constituents as the connotations could be subtle yet significant. When employing any post-manufacturing thermal treatment to a biomaterial, equal consideration should be given to the biological ramifications as that given to the material's mechanical properties.

\section{$\underline{\text { Acknowledgements }}$}

We would like to thank the EPSRC-CASE award with Smith \& Nephew for the funding and supporting LAD. The authors would also like to thank Dr. Steve Hinder for his assistance with the XPS analysis. 


\section{References}

[1] Cawley J, Metcalf JEP, Jones AH, Band TJ, Skupien DS. A tribological study of cobalt chromium molybdenum alloys used in metal-on-metal resurfacing hip arthroplasty. Wear. 2003;255:999-1006.

[2] McMinn D. Modern hip resurfacing. London: Springer; 2009.

[3] Kinbrum A, Unsworth A. The wear of high-carbon metal-on-metal bearings after different heat treatments. P I Mech Eng H. 2008;222:887-95.

[4] Tirrell M, Kokkoli E, Biesalski M. The role of surface science in bioengineered materials. Surf Sci. 2002;500:61-83.

[5] Puleo DA, Nanci A. Understanding and controlling the bone-implant interface.

Biomaterials. 1999;20:2311-21.

[6] Kasemo B, Gold J. Implant surfaces and interface processes. Adv Dent Res. 1999;13:820.

[7] Rattana A, Abel ML, Watts JF. ToF-SIMS studies of the adsorption of epoxy resin molecules on organosilane-treated aluminium: Adsorption kinetics and adsorption isotherms. Int J Adhes Adhes. 2006;26:28-39.

[8] Bailey R, Castle JE. XPS Study of Adsorption of Ethoxysilanes on Iron. J Mater Sci. 1977;12:2049-55.

[9] Abel ML, Chehimi MM, Brown AM, Leadley SR, Watts JF. Adsorption-Isotherms of Pmma on a Conducting Polymer by Tof-Sims. J Mater Chem. 1995;5:845-8.

[10] Watts JF, Castle JE. The determination of adsorption isotherms by XPS and ToF-SIMS: their role in adhesion science. Int J Adhes Adhes. 1999;19:435-43.

[11] Aeimbhu A, Castle JE, Singjai P. Accounting for the size of molecules in determination of adsorption isotherms by XPS; exemplified by adsorption of chicken egg albumin on titanium. Surf Interface Anal. 2005;37:1127-36.

[12] Salvi AM, Castle JE, Watts JF, Desimoni E. Peak Fitting of the Chromium 2p XPS Spectrum. Appl Surf Sci. 1995;90:333-41.

[13] Lowe C, Watts JF, Tegen N, Brown AM. Investigating the adsorption of components of an epoxy primer on to galvanised steel using ToF-SIMS. Surf Coat Int Pt B-C. 2003;86:291300 .

[14] Watts JF, Leadley SR, Castle JE, Blomfield CJ. Adsorption of PMMA on oxidized Al and Si substrates: An investigation by high-resolution X-ray photoelectron spectroscopy. Langmuir. 2000;16:2292-300. 
[15] Serro AP, Gispert MP, Martins MCL, Brogueira P, Colaco R, Saramago B. Adsorption of albumin on prosthetic materials: Implication for tribological behavior. J Biomed Mater Res A. 2006;78A:581-9.

[16] Wimmer MA, Loos J, Nassutt R, Heitkemper M, Fischer A. The acting wear mechanisms on metal-on-metal hip joint bearings: in vitro results. Wear. 2001;250:129-39. [17] Tateiwa T, Clarke IC, Shirasu H, Masaoka T, Shishido T, Yamamoto K. Effect of low protein concentration lubricants in hip simulators. J Orthop Sci. 2006;11:204-11. 


\begin{tabular}{|l|l|}
\hline Element & Nominal compositions of major elements (Mass \%) \\
\hline Chromium & $27-30$ \\
\hline Molybdenum & $5-7$ \\
\hline Nickel & 1 maximum \\
\hline Iron & 0.75 maximum \\
\hline Carbon & 0.35 maximum \\
\hline Manganese & 1 maximum \\
\hline Silicone & 1 maximum \\
\hline Cobalt & Balance \\
\hline
\end{tabular}

Table 1. Chemical compositions as set out by ASTM-F75 98 and ISO 5832 Part 4.

\begin{tabular}{|l|c|c|c|c|c|c|}
\cline { 2 - 7 } \multicolumn{1}{c|}{} & \multicolumn{2}{c|}{ AC } & \multicolumn{2}{c|}{ SA } & \multicolumn{2}{c|}{ HIPSA } \\
\cline { 2 - 7 } \multicolumn{1}{c|}{} & $\begin{array}{c}\text { Surface } \\
\text { concentration (at. \%) }\end{array}$ & SD & $\begin{array}{c}\text { Surface concentration } \\
\text { (at. \%) }\end{array}$ & SD & $\begin{array}{c}\text { Surface concentration } \\
\text { (at. \%) }\end{array}$ & SD \\
\hline C & 33.6 & 4.7 & 34.3 & 4.4 & 33.8 & 2.6 \\
\hline O & 41.0 & 3.5 & 40.1 & 5.4 & 38.9 & 3.8 \\
\hline Cr & 14.3 & 0.4 & 11.7 & 1.8 & 12.5 & 1.9 \\
\hline Co & 10.2 & 2.0 & 13.3 & 2.8 & 14.2 & 4.4 \\
\hline Mo & 0.9 & 0.5 & 0.6 & 0.1 & 0.6 & 0.0 \\
\hline
\end{tabular}

Table 2. Compositions of the clean Co-Cr-Mo ASTM-F75 samples.

\begin{tabular}{|l|c|c|c|}
\cline { 2 - 4 } \multicolumn{1}{c|}{} & $\mathbf{R}^{\mathbf{2}}$ for Langmuir & $\mathbf{R}^{2}$ for Freundlich & $\mathbf{R}^{2}$ for Temkin \\
\hline AC & 1.00 & 0.63 & 0.80 \\
\hline SA & 1.00 & 0.86 & 0.98 \\
\hline HIPSA & 1.00 & 0.84 & 0.84 \\
\hline
\end{tabular}

Table 3. Comparison of the least square values $\left(R^{2}\right)$ for isotherms fitted to the Langmuir, Temkin, and Freundlich model isotherms.

\begin{tabular}{|l|c|c|c|}
\cline { 2 - 4 } \multicolumn{1}{c|}{} & b & $\Gamma \mathrm{m}$, formula value (atomic \%) & $\Gamma \mathrm{m}$, experimental value (atomic \%) \\
\hline AC & 480.7 & 9.8 & 10.2 \\
\hline SA & 14.5 & 10.4 & 10.5 \\
\hline HIPSA & 23.9 & 10.3 & 10.7 \\
\hline
\end{tabular}

Table 4. Theoretically and experimentally derived values for $\mathrm{b}$ and $\mathrm{\Gamma m}$. 


\section{Captions}

Figure 1. XPS survey spectrum for a cleaned and polished AC sample.

Figure 2. High resolution fitted XPS spectra of Cr2p region.

Figure 3. XPS survey spectrum for AC sample in 100\% v/v FBS.

Figure 4. XPS spectra from N1s region plotted on an arbitrary scale to show the relative intensities obtained after immersion in a series of FBS concentrations.

Figure 5. The surface concentration of nitrogen and chromium as a result of protein adsorption on the AC sample, plotted as a function of FBS concentration.

Figure 6. The surface concentration of nitrogen and chromium as a result of protein adsorption on the SA sample, plotted as a function of FBS concentration.

Figure 7. The surface concentration of nitrogen and chromium as a result of protein adsorption on the HIPSA sample, plotted as a function of FBS concentration.

Figure 8. Adsorption isotherm based on the surface concentration of nitrogen presented on a) Linear scale and b) Logarithmic scale.

Figure 9. Adsorption isotherm based on the surface concentration of chromium presented on a) Liner scale b) Logarithmic scale.

Figure 10. Magnified portion of adsorption isotherm showing differences in 'knee' shape between the three samples. 


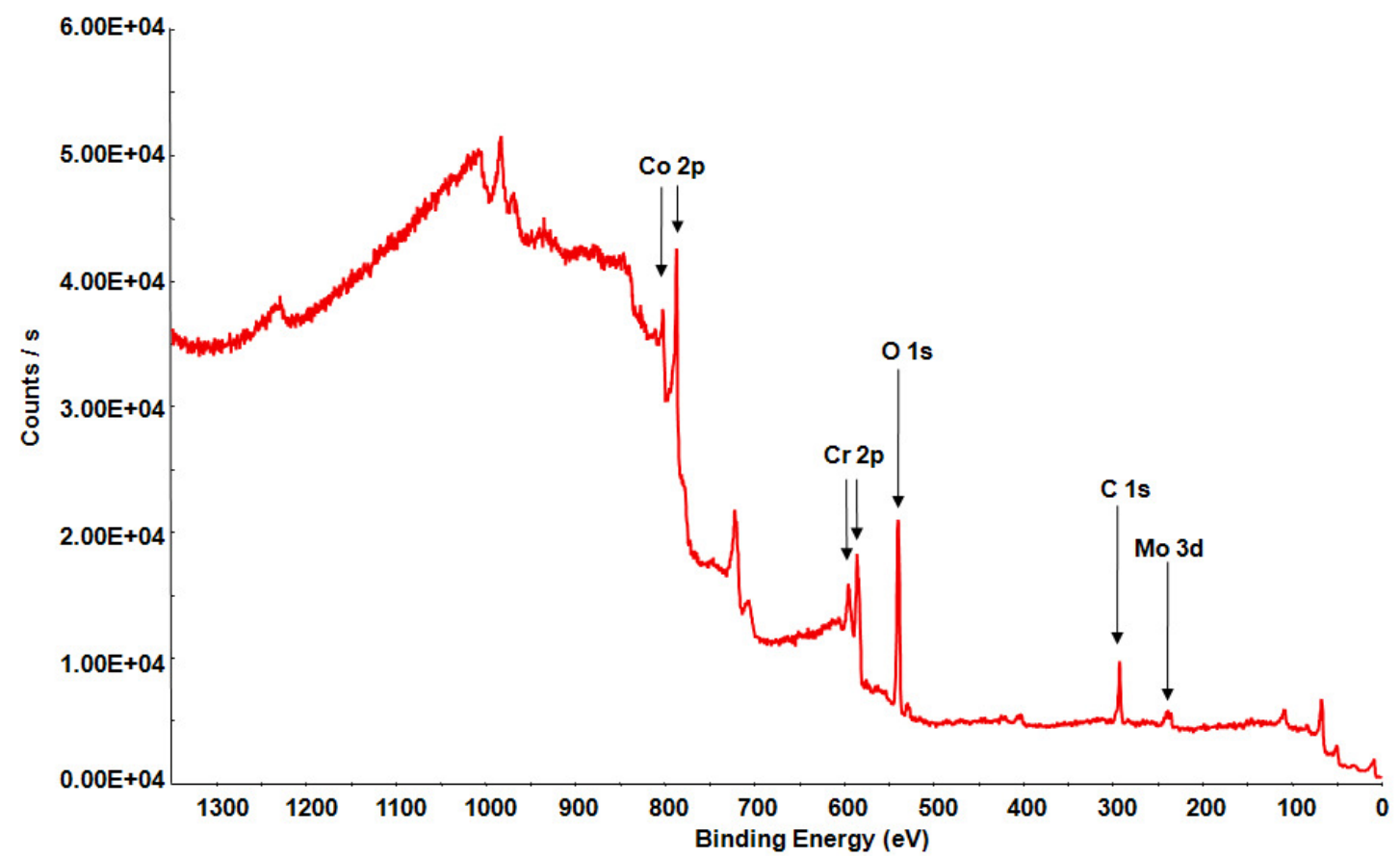

Figure 1. XPS survey spectrum for a cleaned and polished AC sample.

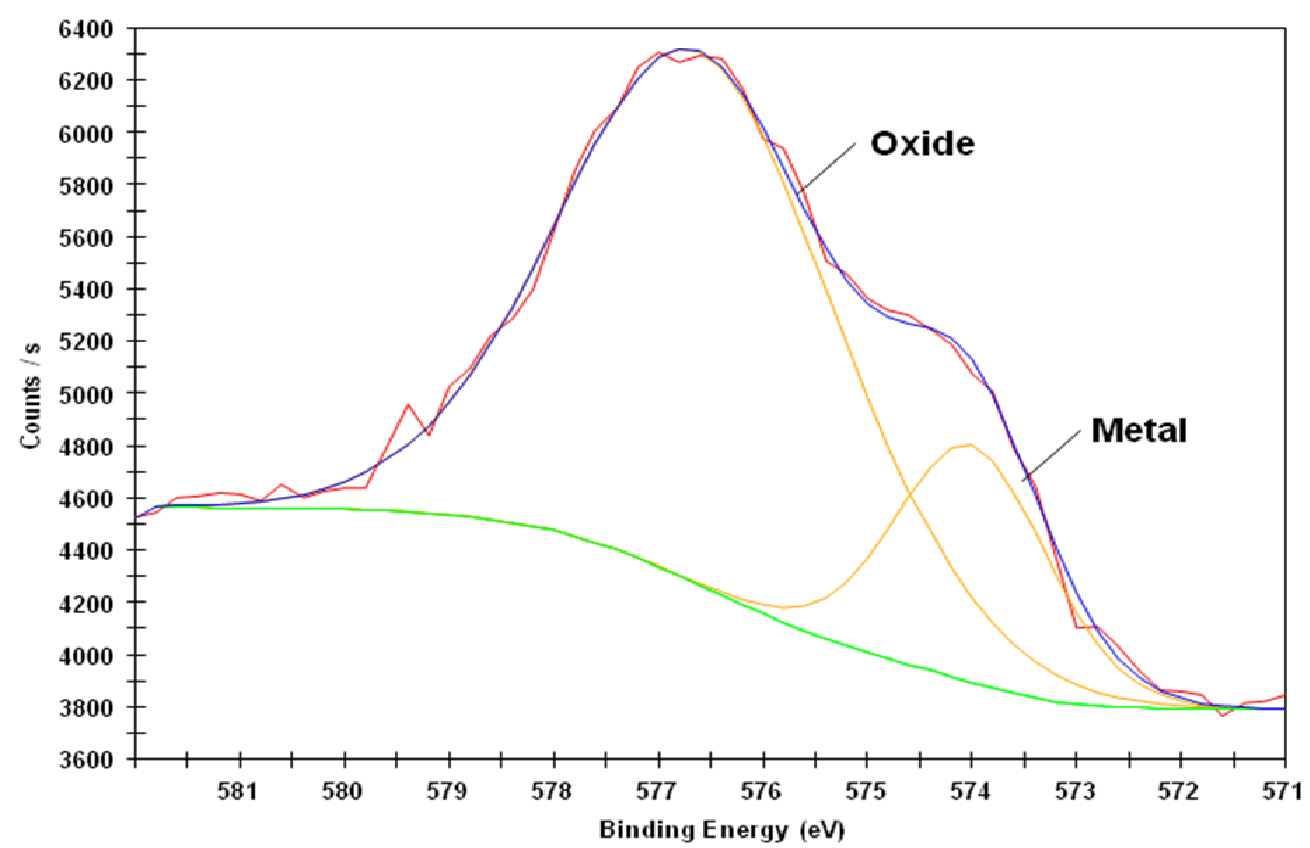

Figure 2. High resolution fitted XPS spectra of Cr2p region. 


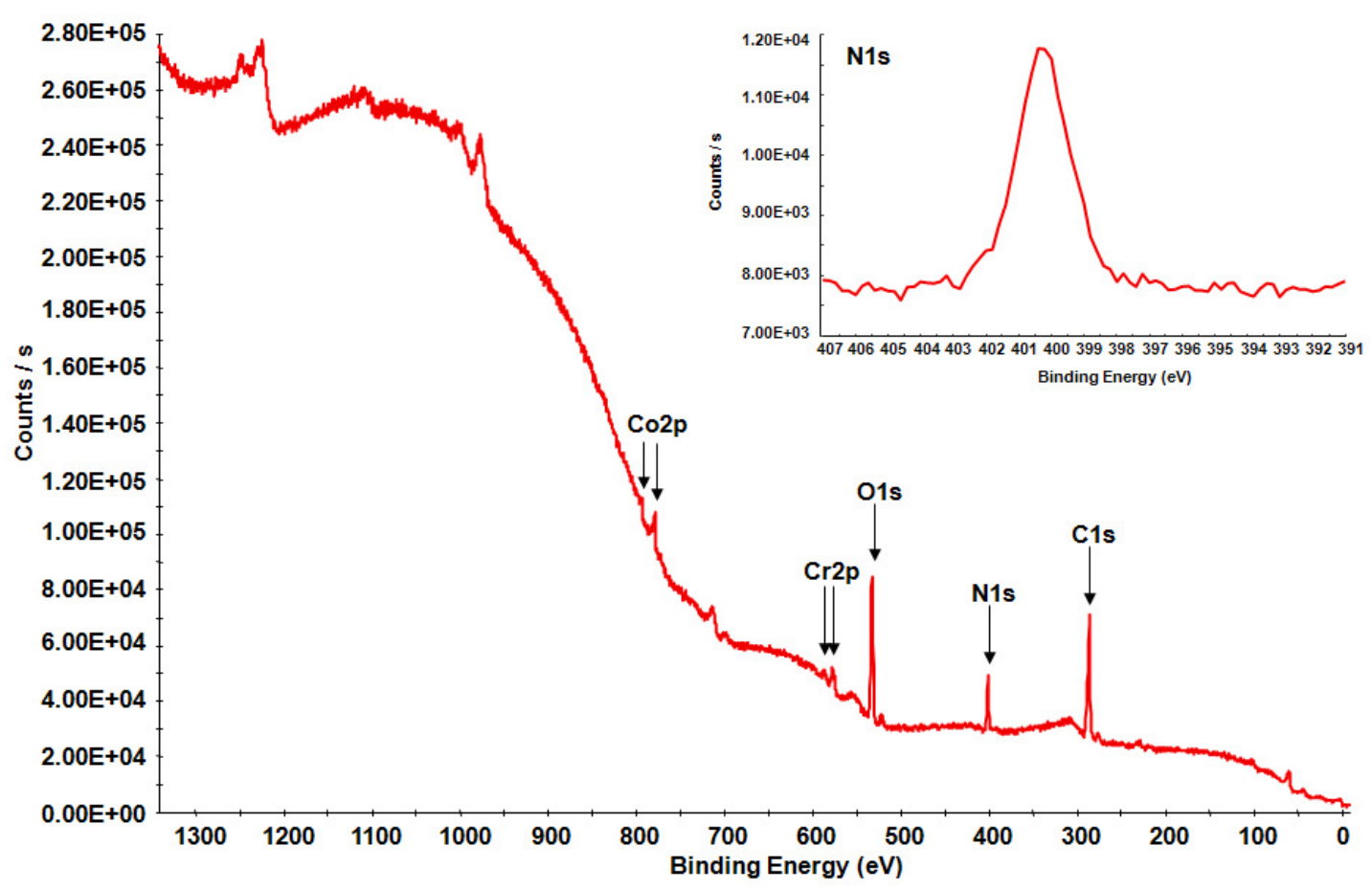

Figure 3. XPS survey spectrum for AC sample in $100 \% \mathrm{v} / \mathrm{v}$ FBS.

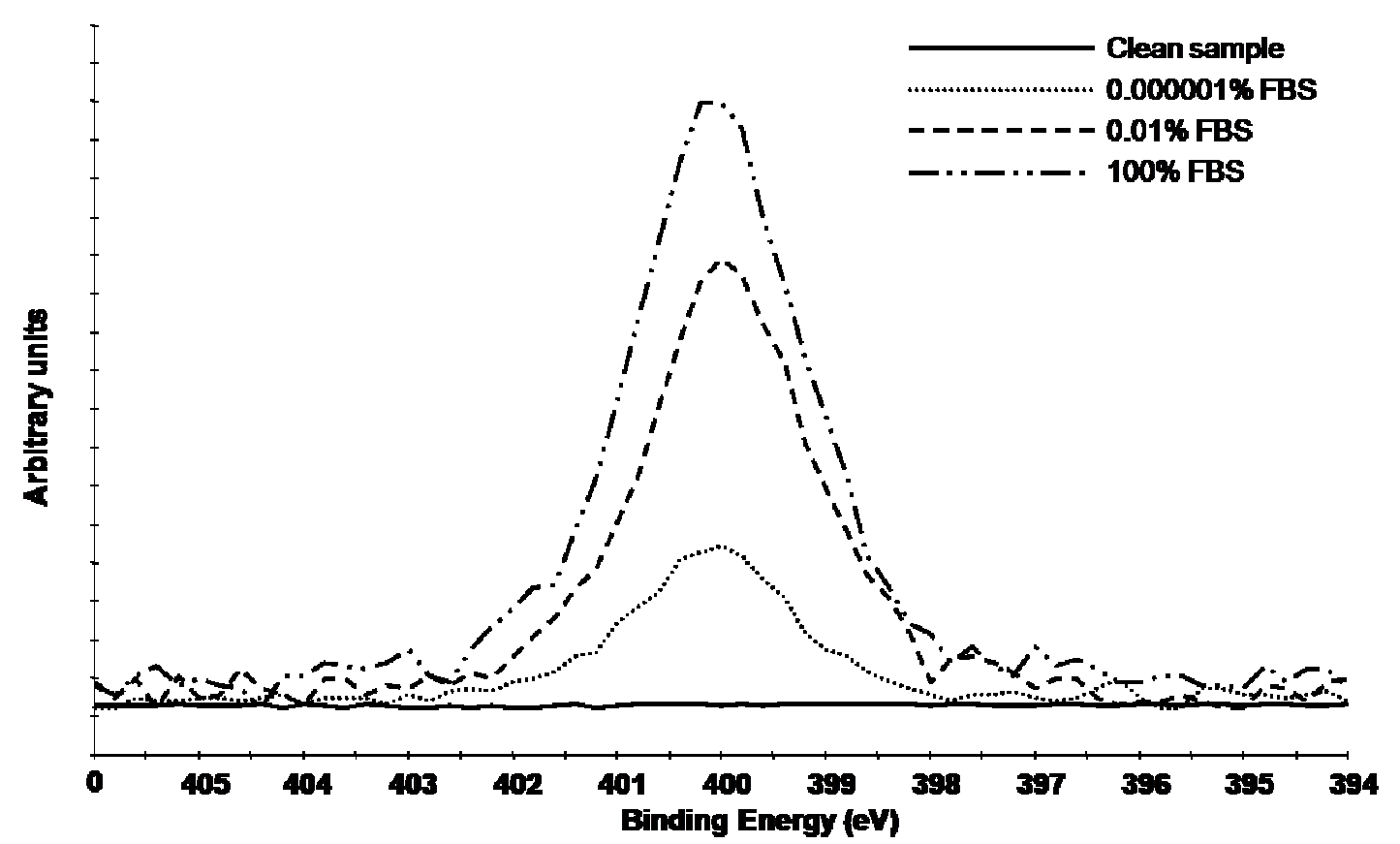

Figure 4. XPS spectra from N1s region plotted on an arbitrary scale to show the relative intensities obtained after immersion in a series of FBS concentrations. 


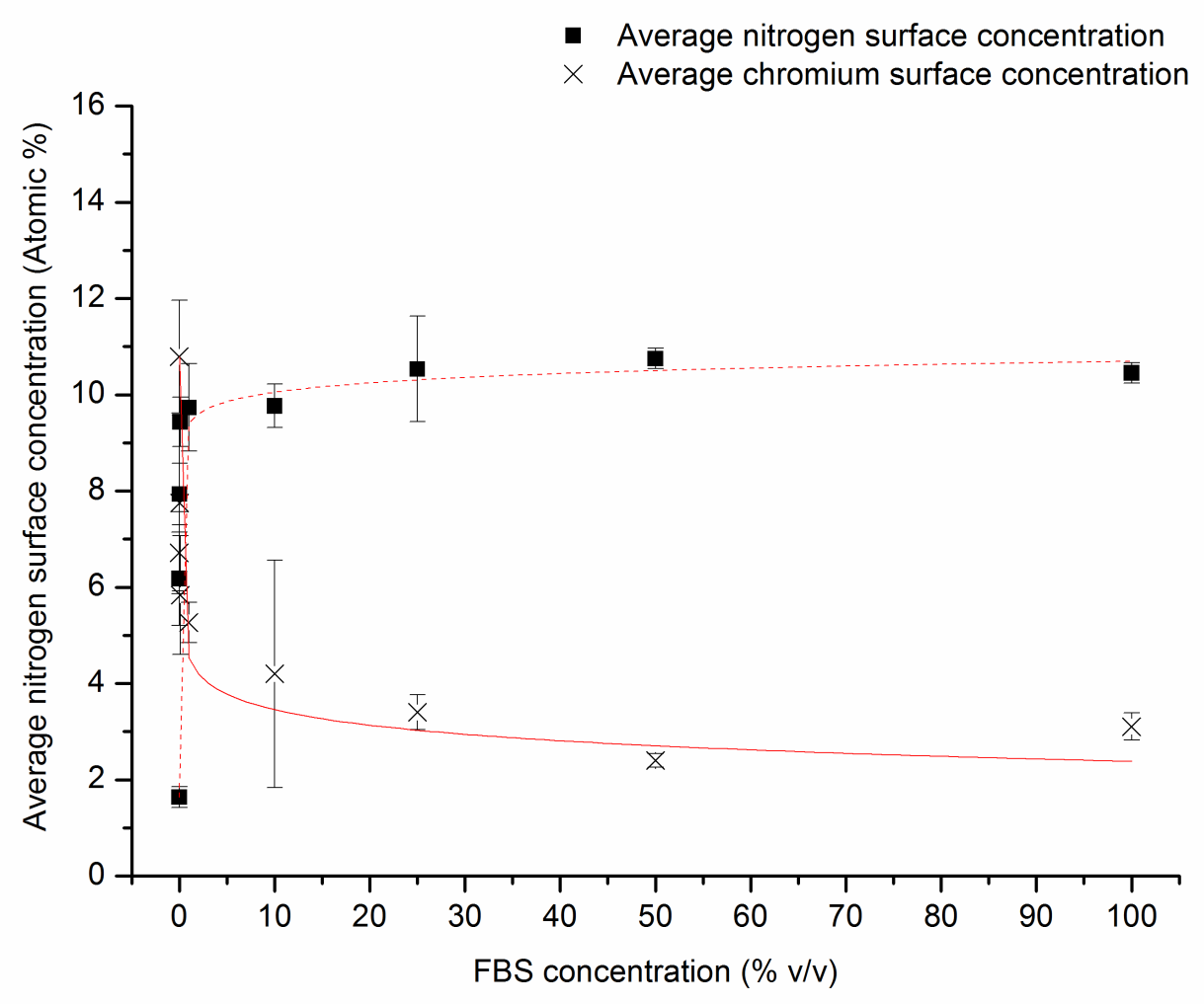

Figure 5. The surface concentration of nitrogen and chromium as a result of protein adsorption on the AC sample, plotted as a function of FBS concentration.

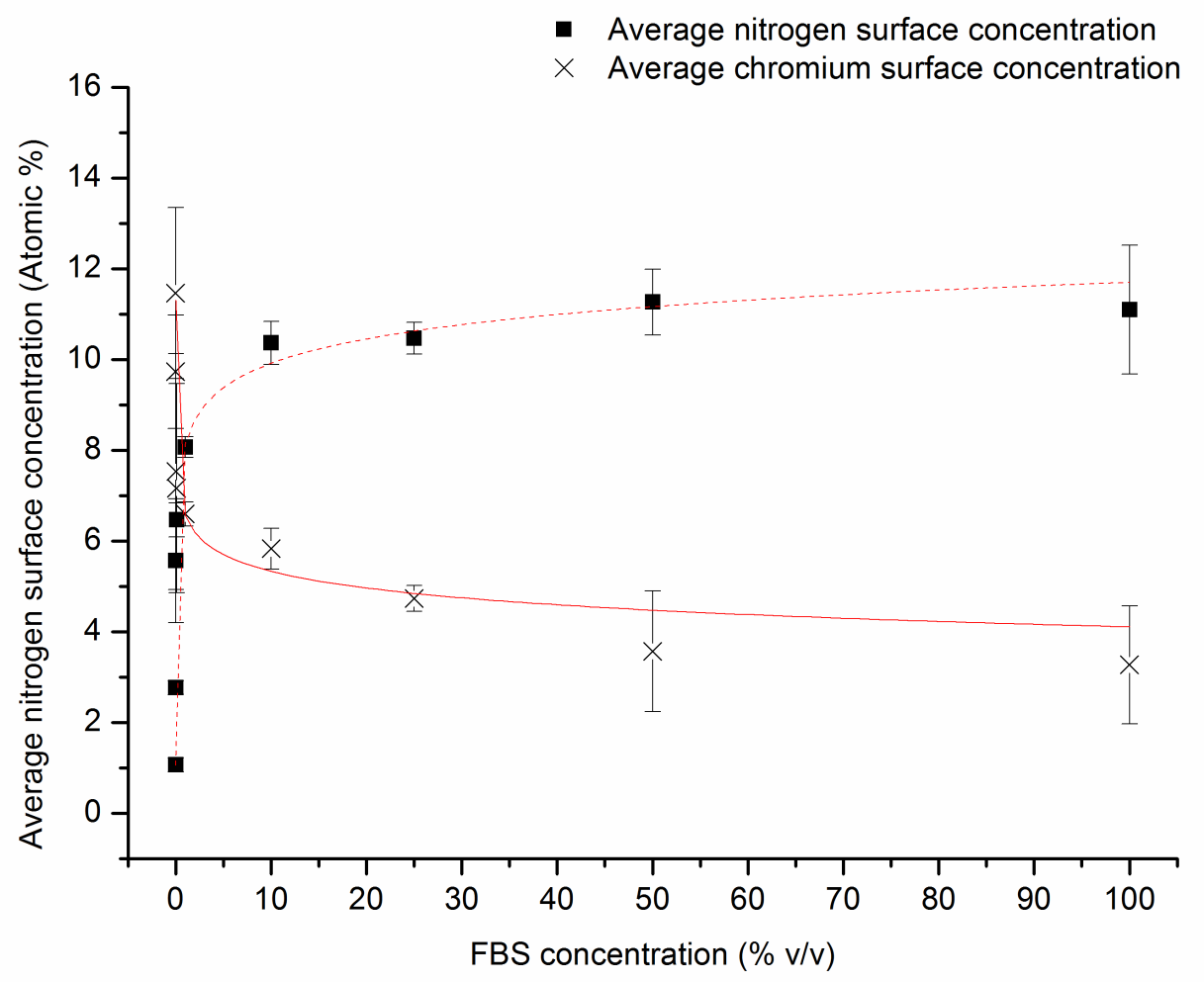

Figure 6. The surface concentration of nitrogen and chromium as a result of protein adsorption on the SA sample, plotted as a function of FBS concentration. 
- Average nitrogen surface concentration

$\times$ Average chromium surface concentration

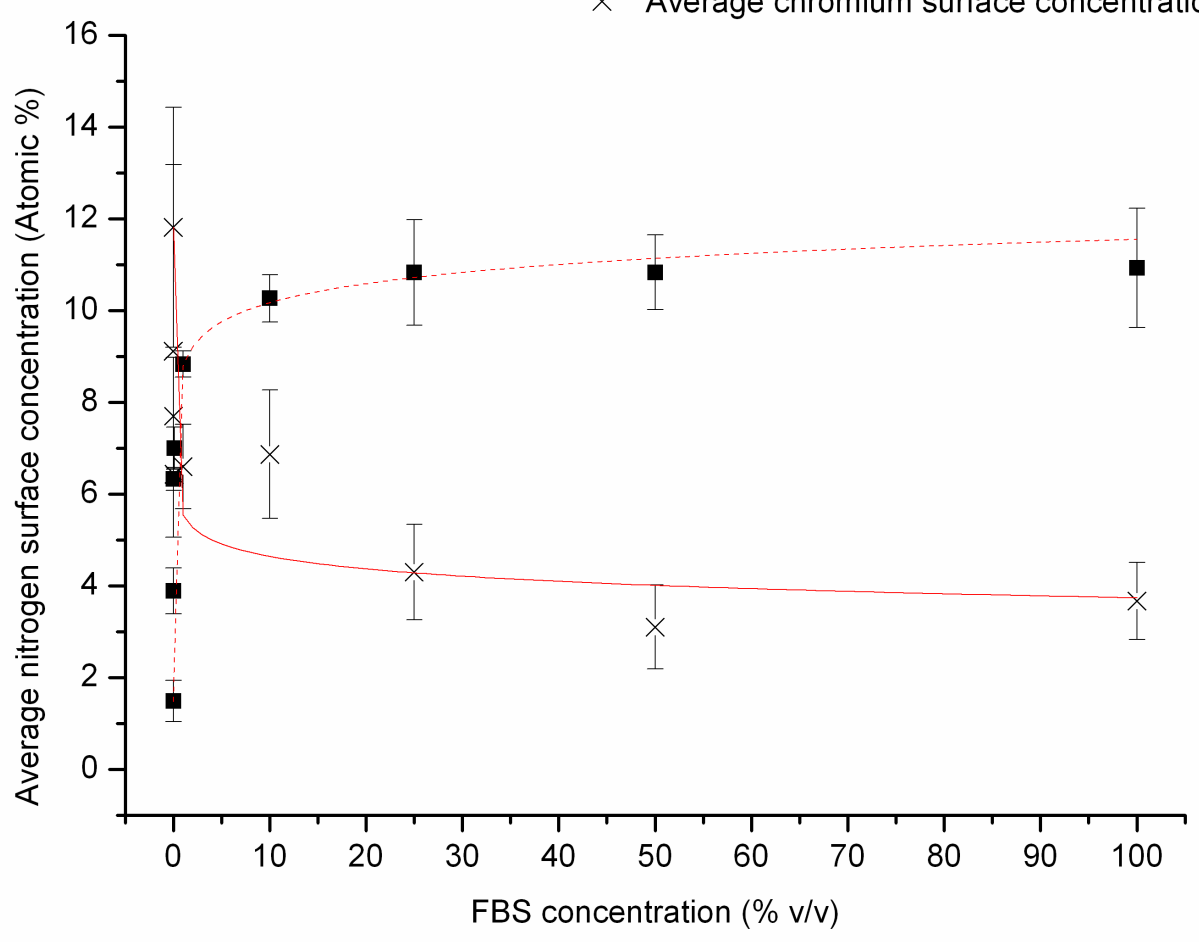

Figure 7. The surface concentration of nitrogen and chromium as a result of protein adsorption on the HIPSA sample, plotted as a function of FBS concentration. 

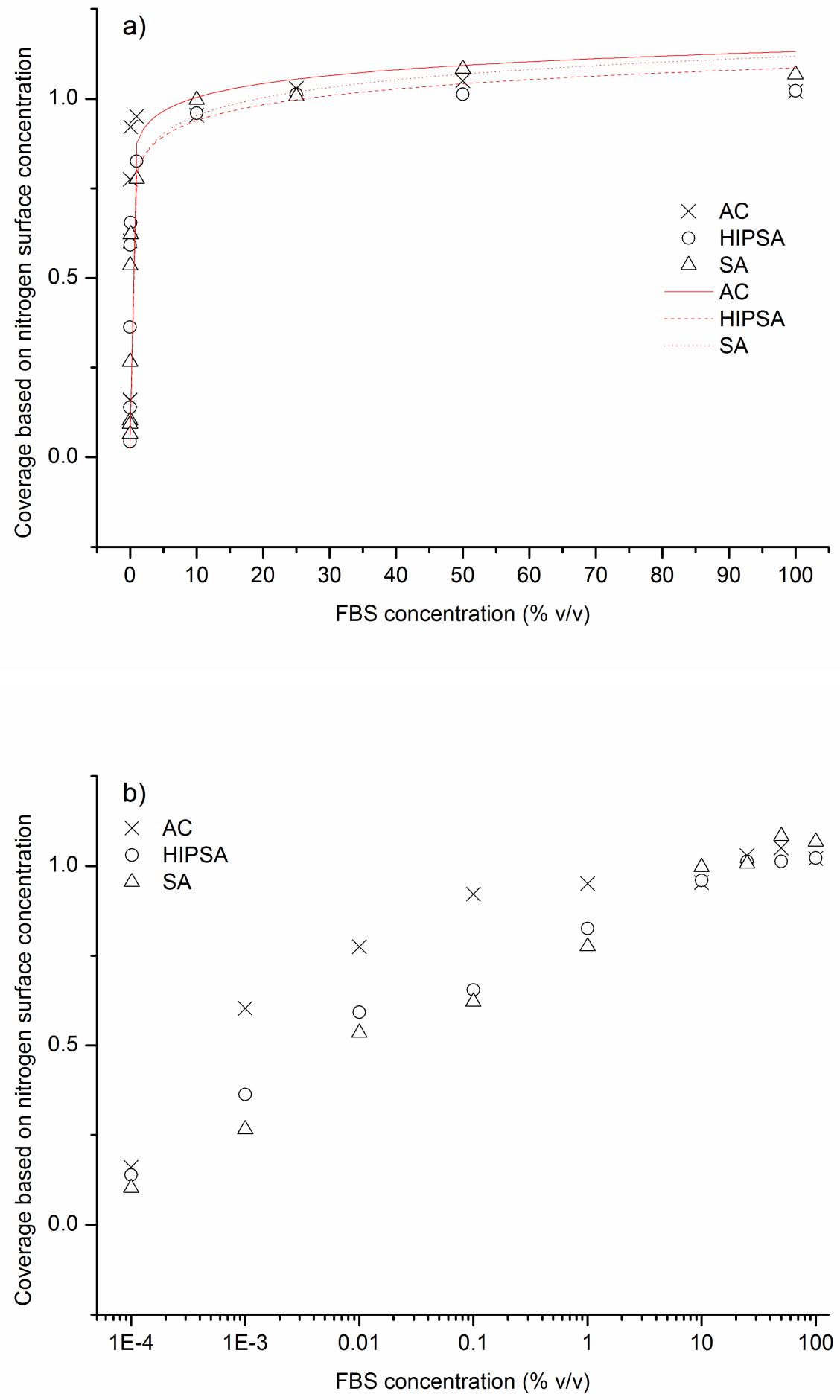

Figure 8. Adsorption isotherm based on the surface concentration of nitrogen presented on a a) Linear scale and a b) Logarithmic scale. 

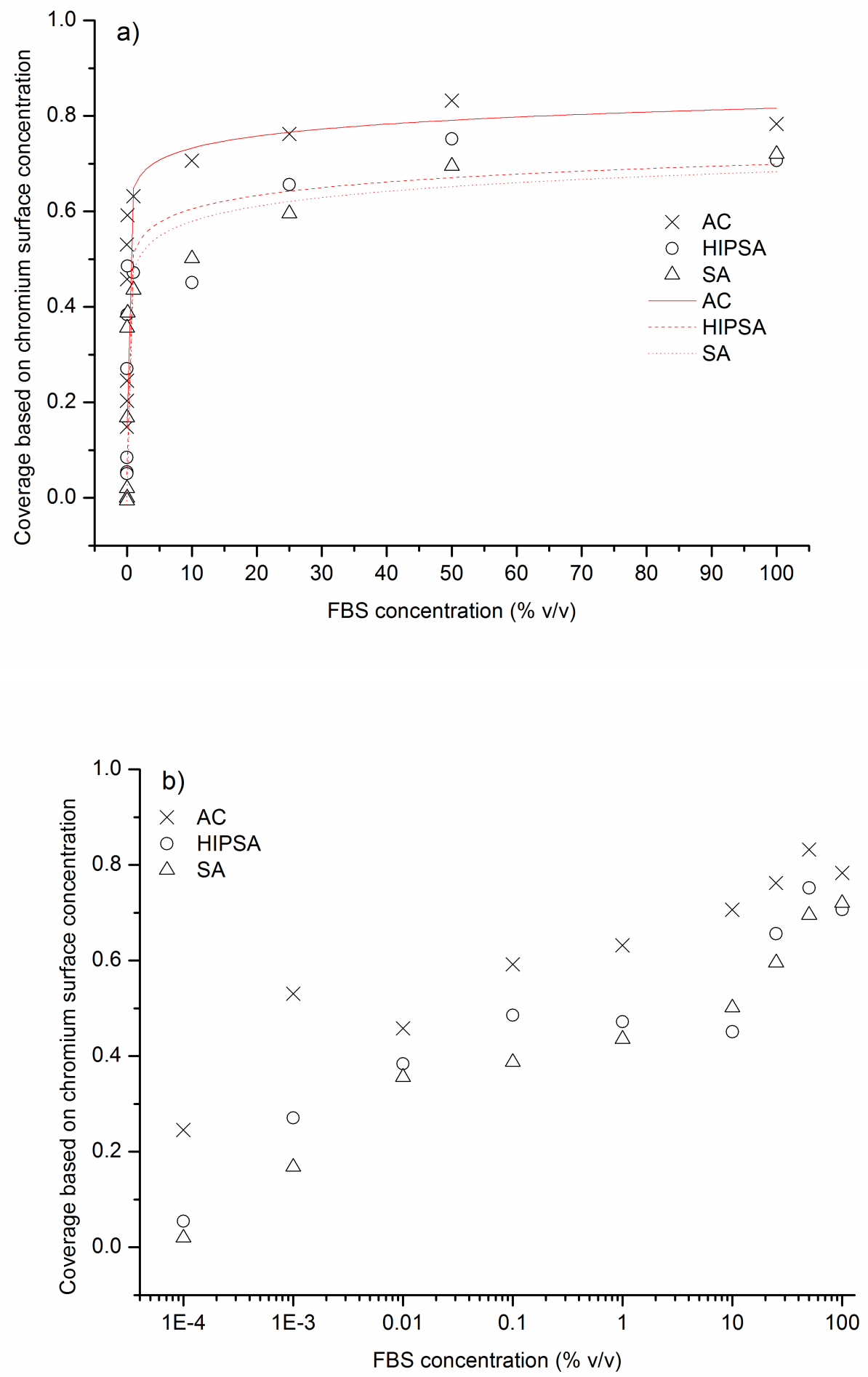

Figure 9. Adsorption isotherm based on the surface concentration of chromium presented on a) Liner scale b) Logarithmic scale. 


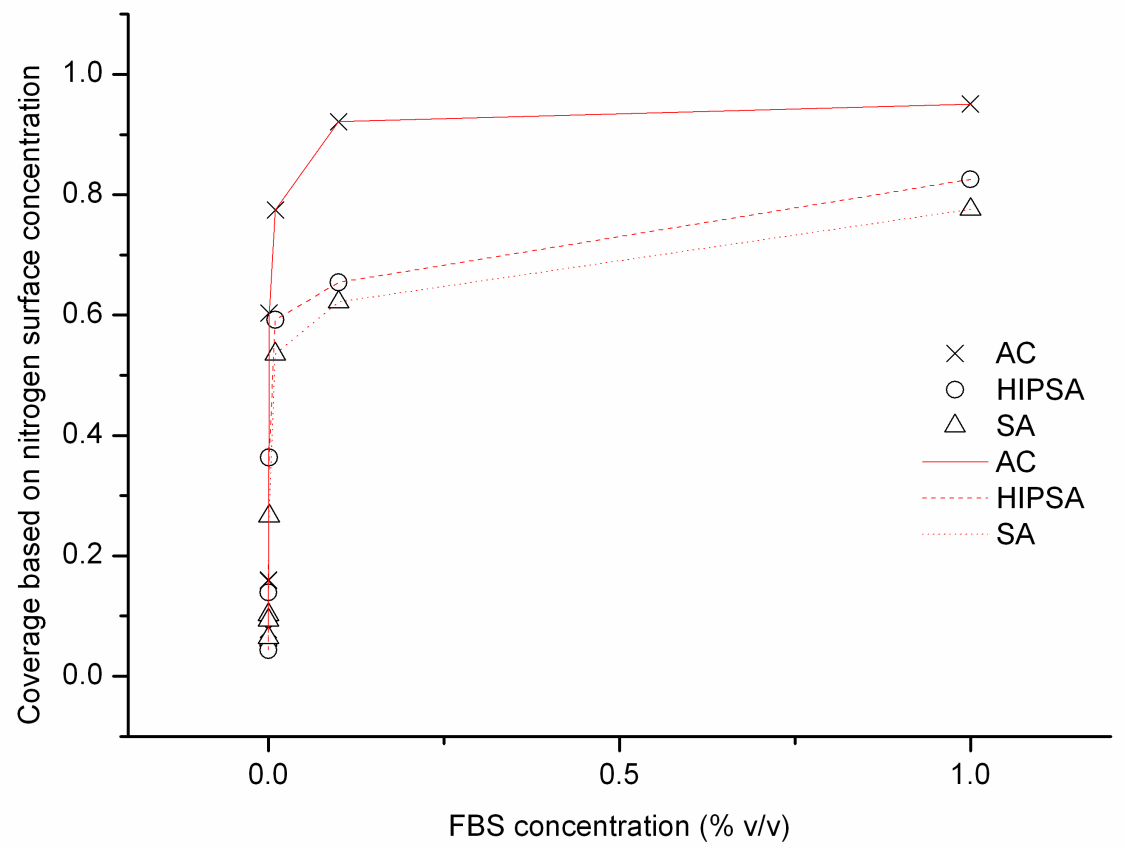

Figure 10. Magnified portion of adsorption isotherm showing differences in 'knee' shape between the three samples. 